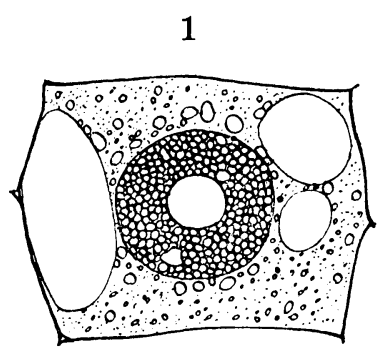

7

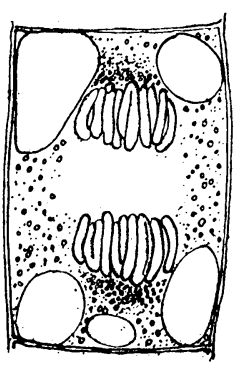

3

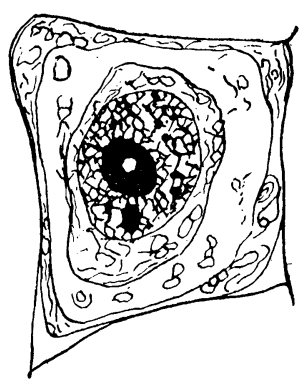

5
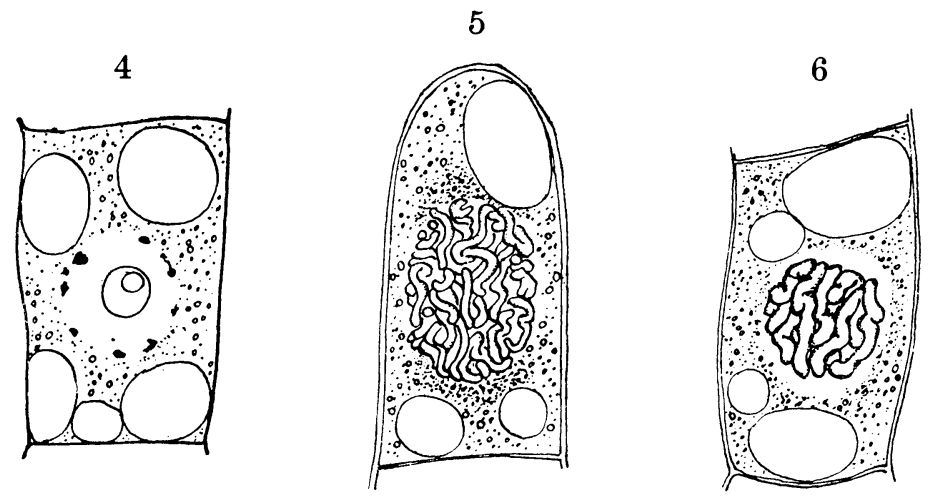

2
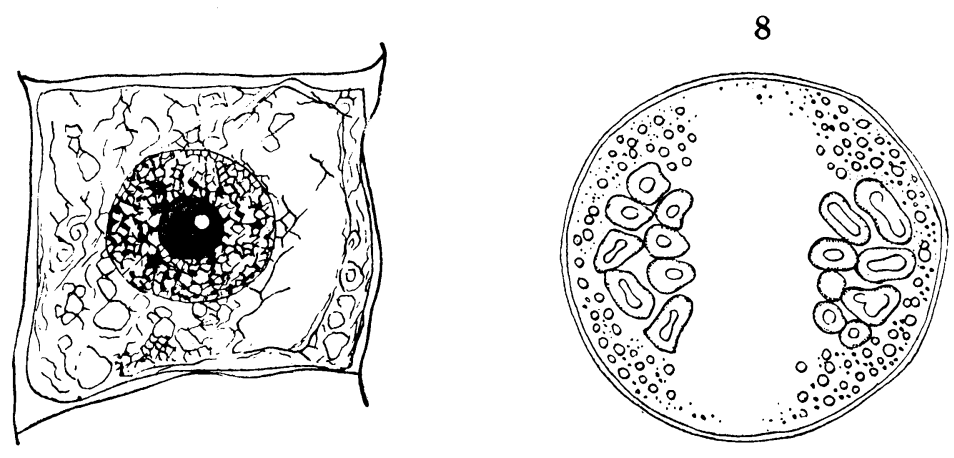

Yamaha del.

YАMАHA-Lebendbeobachtung 


\title{
Über die Lebendbeobachtung der Zellstrukturen, nebst dem Artefaktproblem in Pflanzenzytologie
}

\author{
Contributions to Cytology and Genetics from the Departments of Plant- \\ Morphology and of Genetics, Botanical Institute, Faculty of \\ Science, Tokyo Imperial University, No. 55
}

von

\section{G. Yamaha}

Hierzu Tafel II

In den Naturwissenschaften spielt die Methode der Untersuchung eine wichtige Rolle bei der Kritik der Ergebnisse derselben. Die nähere Bemerkung der Technik gehört deshalb unbedingt in die Darstellung der Untersuchung. Jedoch wird bei der kritischen Verwertung des Resultates die Kunstgerechtigkeit im allgemeinen stillschweigend angenommen. So können wir in diesem Punkt nicht allzu sorgfältig verfahren.

Während die Mikrotomtechnik, welche seit den achtzigen Jahren des letzten Jahrhunderts in die Pflanzenzytologie eingebürgert worden ist, uns einerseits reiche Kenntnisse über die Einzelheiten der Zellstrukturen erschaffen hat, gibt sie andererseits einzelnen Forschern nicht selten Veranlassung, sich gewissen Täuschungen hinzugeben, indem sich bei der Vorbehandlung, besonders bei der Fixierung des Materials, deren sie benötigt, eine Reihe von Artefakten mitten unter wahre Strukturelemente einschmuggeln. Damals, wo neben den lebenden Objekten auch die fixierten zur Anwendung gelangten, scheinen sich Zytologen über die Brauchbarkeit der letzteren ziemlich skeptisch benommen $\mathrm{zu}$ haben, so dass sie sich keine Mühe verdriessen liessen, jeweils lebende Objekte mit in Vergleich zu ziehen (vgl. Tischler 
('2 I-22, S. 236), Lundegårdh ('22, S. 228)). Später mit dem Fortschritte der mikroskopischen Technik ging aber allmählich die allgemeine Tendenz der Zytologen dahin, sich ausschliesslich mit fixiertem Material zu beschäftigen. Trotz des Aufsehens, das die sinnreichen Experimente von Fischer, Berg, HARdy und ManN seinerzeit erregten, scheint man doch gegenwärtig auf zytologischem Gebiete bezüglich der Zuverlässigkeit verschiedener Fixierungsbilder einem Optimismus zu huldigen (vgl. Lidforss ('I 5, S. 233), LUNDEGÅRDH ('22, S. 228), Wilson ('24, S. 4) usw.). Auffallend ist, dass bei der Mehrheit der zytologischen Arbeiten, wie sie während der ersten zwei Dezennien des jetzigen Jahrhunderts ins Feld geführt worden sind, man immer wieder lediglich auf das einerlei Fixierungsmittel, FlemMINGs Lösung, gebaut hat.

Je näher man sich auf die Einzelheiten der Protoplasmastrukturen einlassen will, desto mehr wird es darauf ankommen, eine genaue Scheidung zwischen Natur und Kunst zu treffen, was sich jedoch nicht so leicht durchführen lässt, wie es zunächst scheint. In letzter Beziehung sind bisher zwei Wege eingeschlagen worden, nämlich erstens die direkte Beobachtung an lebenden Objekten und zweitens die vergleichenden Untersuchungen der verschieden fixierten Bilder. Der erste wäre der nächstliegende und sicherste, abgesehen davon, dass er mit mehreren technischen Schwierigkeiten behaftet ist, und zwar u.a. schwere Unterscheidung einzelner lebenden Strukturelemente voneinander, wegen ihrer geringen Differenz der Brechungszahlen und ihrer Farblosigkeit, ferner leichte Zerstörbarkeit der lebenden Strukturen des Protoplasmas. Ich betone hier deshalb die beiden am meisten bekannten Tatsachen, weil es eben diese Eigentümlichkeiten sind, welche die Vitalität der Zellen kennzeichnen, und welche weiter der Lebendbeobachtung die bisher leicht unterschätzte hohe Bedeutung beimessen, dass man nämlich imstande ist besonders $\mathrm{zu}$ ermitteln, wie und inwieweit einzelne Zellbestandteile beim Zellentode ihre kolloidalen Zustände, z.B. Hydratationsgrad, Dispersitätsgrad, Dispersionsweise usw. verändern können.

Was das erste Moment anbelangt, so leistet die Dunkelfeldbeleuchtung vieles, zur Erleichterung der Lebendbeobachtung vornehmlich mittels eines Wechselkondensors, welcher das jeweilige schnelle Abwechseln zwischen Hell- und Dunkelfeld gestattet, falls die zur Beobachtung herangezogene Zelle ziemlich dünn behäutet und 
nicht so dicht mit Einschlüssen beladen ist. Bei meiner Beobachtung wurde ein mit einer ausklappbaren Zentralblende ("expanding stop ") versehener holoskopischer Ölimmersionskondensor (N.A. I,4) WaTsons benutzt. Damit man dabei die Zellen möglichst lang gesund erhalten könne, müssen folgende Vorsichtsmassregeln eingehalten werden:

I. Die Zellen müssen selbst vor dem leisesten mechanischen Druck (z.B. Messerdruck beim Schneiden, Deckglasbelastung usw.) bewahrt werden. ${ }^{1)}$

2. Man darf das Objekt fortgesetzt nicht länger als einige Minuten dem starken Lichte (Lichtquelle des Mikroskops) aussetzen.

3. Es empfiehlt sich, namentlich dann dringend, wenn die Beobachtung länger als eine halbe Stunde fortgeführt werden soll, das im destillierten Wasser gelöste Kohlensäuregas möglichst zu verdrängen, so dass die Reaktion desselben tunlichst der neutralen $(\mathrm{PH}=7)$ näher kommt.

4. Die Isotonie der Beobachtungsflüssigkeit darf nicht durch Zusatz von Elektrolyten (z.B. Kochsalz, Salpeter, Harnstoff usw.) hergestellt werden. Auch das Hühnereiweiss und die Gewebeflüssigkeit aus derselben Pflanze haben sich als Beobachtungsmittel nicht ganz einwandfrei herausgestellt.") Als Zusatzmittel machte ich immer vom chemisch reinen Rohrzucker Gebrauch und zwar nach Ausprobieren in folgenden Konzentrationen; 3-5 proz. für Haarzellen, 2-3 proz. für Wurzelzellen, 5-10 proz. für Pollenmutterzellen. (Vgl. Schaede '25, S. 232).

5. Ich habe in meisten Fällen die Lebendbeobachtung an ein und demselben Material nicht länger als eine Viertelstunde fortgesetzt, zumal es sich um die Pollenmutterzellen und Wurzelspitzenzellen handelte, bei welchen ich schon binnen dieser kurzen Zeit eine Reihe von unverkennbaren nekrobiotischen Veränderungen nicht selten eintreten sah, was allem Anschein nach auf die Beschädigung der Zellen beim Herauspräparieren zurückzuführen ist.

Es ist nicht einzusehen, dass nach Schaede ('25, S. 232, 236 usw.) das gewöhnliche destillierte Wasser (bei unsrigem $\mathrm{PH}_{\mathrm{H}}=5,2-5,4$ wegen gelösten Kohlensäuregases) ebenso giftig wirken soll wie das

I) Die Labilität der Protoplasmastrukturen gegenüber mechanischen Eingriffen ist schon mehrfach hervorgehoben. Vgl. z. B. Lepeschkin ('24).

2) Vgl. Schwarz ('92, S. 53), Befr ('o6, S. 299) usw: 
Leitungswasser (bei unsrigem $\mathrm{P}_{\mathrm{H}}=7,2-7,4$ ), welch letzteres sich als Beobachtungsmittel erfahrungsgemäss häufig viel geeigneter erwiesen hat, als das aus Gläsern vorsichtig umdestillierte Wasser (von demselben $\mathrm{PH}_{\mathrm{H}}$ wie das des gewöhnlichen). In diesem Zusammenhange sei hier bemerkt, dass es jedenfalls auch mit allen Kautelen uns nicht gelungen ist, das herausgeschnittene lebende Präparat unter Deckglas so lange ganz gesund zu erhalten, wie es Schaede geschehen $\mathrm{zu}$ sein scheint.

Über die verschiedenen Fixierungsbilder wurde schon anderswo manches besprochen (s. Yamaha '25 a u. b). Übrigens gedenke ich, die Frage in kurzem in einer besonderen Abhandlung noch weiter $z u$ erörtern. Hier sei aber vorläufig hervorgehoben, dass auch auf diesem Wege uns manche Schwierigkeiten entgegentreten, was letzten Endes darauf beruht, dass die Theorie der Fixierungsprozesse zur Zeit noch eine zuverlässige physikalisch-chemische Grundlage entbehrt. In dieser Richtung erhielten wir allerdings schon durch die sinnreichen Untersuchungen von Fischer, Berg, Hardy und ManN in ausgedehntem Masse einen Hinweis. Solche Schlussfolgerung, wie sie hauptsächlich aus den gewonnenen Versuchsergebnissen mit künstlich dargestellten Stoffen (Eiweisskörper, Nukleinsäuren usw.) gezogen worden ist, lässt sich doch nicht ohne Weiteres auf die lebenden Objekte ausdehnen, da man dort immer gezwungen ist, sich von den verschiedenen wichtigen Faktoren abzuschliessen, welche sich bei dem Fixierungsprozesse in den lebenden Zellen geltend machen (s. auch LUNDEGÅRDH 'I2a, S. 233ff).

Bei der vergleichenden Untersuchung der mit verschiedenartigen Fixierungsmitteln erzielten Strukturbilder müssen wir unter anderem folgendes ins Auge fassen:

I. Alle gebräuchlichen Fixierungsmittel wirken auf die lebenden Strukturen in mancher Hinsicht in recht ähnlicher Weise. Daher kann man nur aus der Gleichheit der Bilder bei verschiedener Fixierung nicht immer auf die Präformität derselben schliessen.

2. Es gibt eine nicht geringe Anzahl von unfixierbaren Strukturelementen in der lebenden Zelle, welche deshalb stets in fixiertem Zustande unserer.Bemerkung entgehen (vgl. auch z.B. Kunstler u. Prévost ('24, S. 147), Lepeschkin ('24, S. i6i) usw.).

3. Ein und dasselbe Strukturbild in fixiertem Zustande kann bei der Fixierung der Zellen nicht nur auf verschiedene Weise zur 
Erscheinung kommen, sondern auch völlig ungleichartigen Zellbestandteilen seinen Ursprung verdanken. Um ein Beispiel zu nennen, lassen sich die Vakuolen, wie sie am häufigsten im Zytoplasma lebender Zellen anzutreffen sind, der Regel nach ziemlich schwer fixieren, indem sie bei der Fixierung sehr leicht der Grössen-, Formen- oder Lageveränderung und ferner auch dem gänzlichen Schwund (Devakuolisation Bergs) anheimfallen. Die ganz übereinstimmende Strukturform findet sich nicht selten auch im fixierten Material als eine Artefakte ein, welche beispielsweise durch die lokale Auflösung, die abnorm erhöhte Hydratisierung, usw. des Zytoplasmas entsteht. Ausserdem werden weiterhin auch noch die durch Fixierungsmittel entstellten Plastiden, Cytosomen oder sonstige Zelleinschlüsse den vakuoligen Bau des Zytoplasmas bedingen.

Um den obigen Übelständen abzuhelfen, welche man bei der Beschäftigung mit nur fixierten Zellstrukturen unvermeidlich zur Folge hat, müssen auch lebende Objekte von Zeit zu Zeit sorgfältig zur Beobachtung herangezogen werden, was bislang leicht versäumt worden $z u$ sein scheint. Auf diesem Wege sind wir in der Lage, eine genaue Einsicht in die kolloidalen sowie metakolloidalen Strukturen des Protoplasmas (s. unten) zu gewinnen, wobei auf folgende drei Momente noch eine besondere Rücksicht zu nehmen ist :

I. Es muss immer ein vollauf triftiger Grund vorhanden sein, einer im Leben nicht $z u$ erkennenden und erst nach Fixierung $z u$ Gesicht tretenden Zellstruktur Präformität beimessen zu dürfen. Man wäre nicht dazu berechtigt, bloss auf einem Analogieschlusse zu fussen. Ebensowenig darf die Zierlichkeit der Fixierungsbilder zur alleinigen Folie nicht dazu dienen, dass es sich um eine lebend , unsichtbare Zellstruktur " handeln soll.

2. Eine Anzahl von Strukturelementen, welche sich zwar sowohl im Leben wie auch im fixierten Zustand erkennen lassen, fallen doch beim verwechselnd ähnlichen Aussehen dem Wesen nach voneinander ganz verschieden aus. Es ist häufig der Fall bei granulären, vakuoligen oder membranartigen Strukturen des Protoplasmas.

3. Die nähere Vergleichung des lebenden Materials mit dem fixierten wird noch weiter durch den Umstand erschwert, dass bei der mikroskopischen Untersuchung die beiden methodisch für gewöhnlich miteinander nicht völlig übereinstimmend behandelt werden ; bei der Lebendbeobachtung nämlich sind die ganzen, nicht angeschnitte- 
nen Zellen üblicherweise ungefärbt im Wasser (Brechungsindex 1,333) bespült. ${ }^{\text {) }}$ Dementsprechend richtet sich die Sichtbarkeit einzelner Strukturelemente vorwiegend nach den Verschiedenheiten der Brechungszahlen der letzteren. Bei den Untersuchungen der fixierten Strukturen treten hingegen die Farbennuanzen jeder Zellbestandteile in den Vordergrund, da das Beobachtungsmaterial sich hier auf die vorher künstlich gefärbten, in kanadischem Balsam (Brechungszahl I,547) eingebetteten Zellfragmente bezieht. Es liegt also auf der Hand, dass unter solchen Beobachtungsbedingungen die genaue Identifizierung feiner Strukturen der lebenden Zellen mit denen der fixierten ganz unmöglich ist. Es entspricht gerade dem letzten $Z_{w e c k}$, das fixierte Objekt auch ungefärbt und im Wasser oder weiter in Methylalkohol (Brechungszahl I,330) eingebettet der Besichtigung zu unterziehen.

Für die Auseinanderhaltung der Naturstrukturen von Artefakten haben wir uns noch weiterhin den dritten Weg anzubahnen bestrebt. Mit der Erwartung, dass unter verschiedenen Aussenbedingungen sich die schon im Leben vorhandenen Strukturen von den erst beim oder nach dem Zellentode eintretenden, auch morphologisch übersehbar abweichend kundgeben möchten, wurden eine Reihe von experimentellzytologischen Studien angestellt, indem die Versuchsobjekte (Keimwurzeln, Blütenknospen usw.) entweder lebend verschiedenen abnormen Bedingungen ausgesetzt oder auch durch verschiedene Eingriffe abgetötet wurden. Von den Versuchsergebnissen, die in der Kürze anderswo im Einzelnen behandelt werden sollen, möchte ich im folgenden nur solches vorausschicken, das hierzu in enger Beziehung steht.

I. Streng genommen entsprechen verschiedenartige Fixierungsbilder jedem Stadium der Nekrobiosis der Zellen, welches aber noch nicht so weit vorgerückt ist, dass die metakolloidalen Strukturen (s. unten) schon ununterscheidbar zugrunde gehen würden.

2. Die genannte Nekrobiose der Zellen besteht wesentlich in der irreversiblen Zustandsänderung der Plasmakolloidé, und zwar nach den beiden entgegengesetzten Richtungen hin, d.h. nicht nur nach der

I) Die vitale Färbung wird neuerdings von SCHAfide ('25) in dieser Beziehung empfohlen. Sie kann aber leider nicht bei cien Studien der Kernstrukturen in den Dienst treten. 
absteigenden, z.B. Fällung (Gelbildung, Koagulation), Entmischung usw., sondern auch nach der aufsteigenden, z.B. Peptisation, Gelatinierung, Emulgierung usw. Die absteigende Zustandsänderung scheint häufig, namentlich am leichtesten bei den Strukturen mutmasslich höherer Viskosität, z.B. Zellkern, Spindelsubstanz, Membranstrukturen (s. unten), von dem Umwechseln der Dispersionsweise bzw. Phasenumschlag begleitet $\mathrm{zu}$ sein. Beim Fixierungsprozesse erfahren im übrigen allerlei Strukturen stets mehr oder minder einen Substanzverlust infolge erhöhter Permeabilität (Exosmose). Die letztere, wie sie insbesondere bei der Anwendung der reichlich Säuren oder lipoidlösliche Stoffe (z.B. Alkohole, Ather, Chloroform) enthaltenden Fixiermittel bedeutend hervortritt, kann nicht in geringem Masse zu der Verdeutlichung einzelner Fixierungsbilder, zumal der sog. Membranstrukturen (s. unten, weiter auch Yамана 25a u. b) beitragen.

3. Das erste sichtbare Stadium der eigentlichen Nekrobiosis der Zellen bekundet sich als die aufsteigende Zustandsänderung der Plasmakolloide d.i. Dispersitätsvergrösserung und Hydratisierung, welche die Homogenesierung und begierige Farbstoffaufnahme, wie sich für gewöhnlich bei den allmählich abgestorbenen Zellen erkennen lässt, zur Folge hat. ${ }^{1)}$

Dabei mag das eine oder das andere Strukturelement gänzlich verschwinden, wei es z.B. häufig bei Plastiden, Cytosomen, Mikrosomen, Karyotinkörnern usw. der Fall ist. In demselben Sinne ist zu erklären, der angebliche Schwund der Leukoplasten (nach Miene 'or, S. I 34) und der Verbindungsfäden (nach Schaede '25, S. 24I) beim langsamen Absterben der Zellen.

4. Es nimmt unser besonderes Interesse in Anspruch, dass eine Anzahl der Strukturmodifikationen, welche bei einem Versuchsmaterial nur unter experimentell hergestellten anormalen Bedingungen in die Erscheinung treten, bei einem anderen auch normalerweise wiederkehrend gefunden werden, so z.B. Zurückbleiben des Nukleolus in Metaphase, Ausbleiben der Polkappenbildung, Endverklebung und Quersegmentierung der Chromosomen, mehrpolige Spindelbildung, zentripetale Scheidewandbildung, Karyomerenbildung, usf. Inwieweit diese merkwürdige Tatsache bei der Betrachtung der Artefaktfrage

I) Vgl. auch die Angaben von Lundegånh ('22, S, 280) und Sakamura u. Loo ('25, S. 74). 
eine theoretische Verwertung finden kann, bleibt zur Zeit dahingestellt.

Es hat sich nun zweckmässig herausgestellt, in der Zelle zwei Kategorien der Strukturen voneinander zu unterscheiden, wenn man dabei natürlich auch zwischen beiden keine scharfe Grenzlinie zu ziehen vermag. Beim Zellentode erleidet der feinere Bau einzelner Strukturelemente der Zellen notwendigerweise auch durch Fixiermittel die in einem gewissen Sinne ,, gründliche “ Zerstörung, welche die dabei intrazellular ausgelösten, irreversiblen kolloidalen Veränderungen mit sich bringen, während der gröbere dagegen fernerhin postmortal seinen Bestand erhalten kann. Dem letzteren, dem ich provisorisch die Bezeichnung ,metakolloidal “ beilegen will, und der begrifflich der ,, subkolloidalen “ Struktur früherer Forscher (s.z.B. Ostwald 'I9, S. I 3 Iff) $z \mathrm{u}$ entsprechen scheint, gehören diejenigen Zellbestandteile an, welche bei geeigneter Fixierung, gleichsam unabhängig von ihrem kolloidalen Zustand, dem Äusseren nach leidlich ,, naturgetreu “ gewahrt bleiben. Wenn man also dem Gebrauch nach von der ", guten " oder ,, schlechten “ Fixierung spricht, so sollte es sich stets auf die Strukturen dieser Kategorie beziehen. Hierin sei alles umfasst, dessen Präformität nicht mehr in Frage kommen kann, so z.B. Zellkern, Plastiden, Chondriom, Vakuolen, Plasmastränge, Kerntasche, Karyosom, Chromosomen, Nukleolus usw. Beim näheren Eingehen auf den feineren Bau des Protoplasmas treten uns die Mannigfaltigkeiten der sog. ,, kolloidalen “ Strukturen bzw. Strukturen zweiter Ordnung entgegen, deren Beständigkeit einzig und allein an die eigentümlichen kolloidalen Beschaffenheiten der lebenden Zellen angeknüpft $z u$ sein scheint. Um diese Strukturart drehen sich immer wieder eine Menge von den bisher angefochtenen Artefaktproblemen. Da alle gebräuchlichen Fixiermittel jene kolloidalen Strukturen ja ganz zunichte machen, so sind wir ausserstande, die letzteren als solche in fixiertem Zustand zu Gesicht zu bekommen. Damit soll jedoch nicht gesagt sein, dass sich die morphologischen Studien solcher Fixierungsbilder (A. FIScher '99) keiner Mühe lohnen könnten. Wir würden nur dann fehlgehen, wenn wir in jedem fixierten Strukturelement einen wahren Bau lebenden Protoplasmas erblicken wollten. Die Fixierungsbilder mannigfacher Arten, die alle insofern Artefaktformen darstellen, als man mit den ,, kolloidalen “ Strukturen zu tun hat, müssen vielmehr in einem anderen Sinne verwertet werden, nämlich 
dass sie uns in irgendwelcher Weise von den lebenden Strukturen etwas wiederspiegeln können (vgl. LundeGÂRDH ('I 2a, S. 243, 253 ; '22, S. 228), Ostwald ('I9, S. I42), Bechhold ('20, S. 453) usw.). In diesem Zusammenhange sei hier der Beachtung wert, dass die sog. „Naturgetreuheit " der durch ein Fixiermittel dargebotenen Bilder nicht immer für die Güte desselben ausschlaggebend ist, weil z.B. Osmiumsäure, neutraler Formol usw., wenn sie schon verhältnismässig einwandfreie Naturbilder sowohl des Zytoplasmas wie des Karyoplasmas wiedergeben können, eine zur genauen Beobachtung zarter Zellstrukturen geeignete Fixierung nicht ermöglichen. Da betreffs der ,, kolloidalen “ Strukturen, wie oben bemerkt, von der naturgetreuen Fixierung keine Rede sein kann, so kommt es hier lediglich auf die Verdeutlichung des in Frage kommenden Strukturelements an.

Unter Hinzuziehung des allen obigen soll im folgenden einiges, das ich über kolloidale sowie metakolloidale Strukturen lebender Zellen ermitteln konnte, zusammenfassend dargestellt werden. Der Lebendbeobachtung wurden unterworfen ; Staubfadenhaare von Tradescantia virginica, Schuppenhaare von Farnblättern, Wasserblatthaare von Salvinia natans, Wurzelspitzen von Vicia Faba, Pisum sativum, Allium Cepa, Glycine Soja, Zea Mais, Sagittaria sagittifolia, Ricinus communis, Isoetes japonica, Pollenmutterzellen von Tradescantia virginica, Tricyrtis hirta, Hemerocallis fulva, Lillium speciosum, Yucca gloriosa, Magnolia Kobus, Daphne odora, Bletilla hyacinthina usw.

In den gesund lebenden Zellen schliesst sich das Zytoplasma stets ohne Aussparung dicht an die Innenfläche der Zellwand an. Das Abgelöstsein desselben von der Zellmembran entspricht daher zweifelsohne nicht dem lebenden Zustand der Zellen und tritt auf irreversiblem Wege erst nekrobiotisch oder auch postmortal auf. ${ }^{1)}$ Es ist nämlich entweder auf den Exosmosevorgang infolge erhöhter Permeabilität des Protoplasmas oder auf die nachträgliche Entquellung desselben durch Fixiermittel oder mitunter auch Entwässerungsmittel (Alkohol usw.) zurückzuführen. Die nekrobiotische Zytoplasmaschrumpfung geht unausbleibbar mit der inneren Strukturveränderung des Protoplasmas einher, indem sie einer Reihe von den

I) Vgl. auch ,, Pseudoplasmolyse “ nach Osterhout ('r3) und postmortale bzw. " sekundäre “ Plasmolyse nach Küster ('24, S. 976) und Schneider ('25, S. 37). 
als nekrobiotische Formen oder kürzer Nekroformen bezeichneten Strukturanormalien Ausdruck verleiht. (Ausführliches wird in einer anderen demnächst $z u$ veröffentlichenden Abhandlung vorbehalten). Der im Leben reversibel vorfindlichen Plasmolyse knüpfen sich übrigens einige Strukturmodifikationen der Zellen an.

Abgesehen von der rein physiologischen Seite der Frage, gehen die Ansichten der Forscher noch bis in die jüngste Zeit darüber auseinander, ob sich das Zytoplasma nach Aussen hin auf alle Fälle mit einer auch mikroskopisch auflösbaren Membran (Hautschicht, Plasmamembran usw.) bekleidet (s. z.B. Seifriz ('2 I), Lepeschkin ('24, S. 93ff), Weis ('25) usw.). Nach meiner Erfahrung ist sie einer Gruppe der Strukturtypen zuzurechnen, die ich unter der Bezeichnung „Membranstruktur“ subsumieren will (vgl. auch Yамана '25a u. b). Dazu gehören weiter auch Kernwand, Vakuolenwand, Aussenhülle der Plastiden, Chromosomen usw. Sie stellen alle diejenigen „,kolloidalen " Strukturen dar, welche jeder Phasengrenze des Protoplasmas entsprechen sollen, und zwar die im Leben nicht oder schwer sichtbar sind, die aber beim Zellentode durch Dispersionsverminderung und wahrscheinlich darauffolgenden Phasenwechsel verdeutlicht werden, was weiter durch Exosmose oder Aufösung der Grundsubstanz betreffender Strukturelemente begünstigt werden kann. Sie zeichnen sich dadurch aus, dass sie in lebendem Zustand wenigstens nach Innen hin nicht scharf begrenzt, d.h. nicht doppelt kontouriert erscheinen, wie es jedoch ultramikroskopisch nicht selten auffallen mag (vgl. WeIs '25, S. I48ff). ${ }^{1)}$ Es sei hier noch hervorgehoben, dass sie auch lebend häufig bei etwas weiter herangewachsenen Zellen (z.B. Wurzelspitzen, Haarzellen) vielleicht wegen verminderten Hydratationsgrades mikroskopisch deutlich zu Gesicht gelangen.

Sowohl mikroskopisch wie ultramikroskopisch betrachtet, lassen sich im eigentlichen Zytoplasma, wie hinreichend bekannt, Hyaloplasma (Grundsubstanz einschliesslich optisch leer disperse Phasen) und Mikrosomen (mikroskopisch sowie ultramikroskopisch disperse Phasen) unterscheiden, was unbedingt mit dem kolloidalen Zustand

I) Dasselbe wurde bezüglch der Hautschicht auch früher von verschiedenen Autoren angegeben, so z. B. Kürne (' $64, \mathrm{~S}, 29)$, Hofmpister ('67, S. 3), de Vries ('85, S. 51 I), Pffffer ('21, S. 124), Luningiardir ('22, S. 243), Weis ('25, S. 148) usw. 
der lebenden Zellen zusammenzuhangen scheint. Die beiden stellen also jene ,, kolloidale “ Strukturen dar, und können bei der Fixierung zu der Ausbildung der sog. „, Strukturen “ verschiedener Typen des Zytoplasmas (Fixierungsbilder) beitragen. Bei den sauren Fixiermitteln kommen fernerhin dazu auch die entstellten Plastiden und Chondriosomen hinzu. Die dergestalt entstandenen, gerinnseligen, streifigen, fädigen, filzigen oder netzartigen ,, Strukturen “ vertreten von den beiden lebenden Strukturelementen als solchen nichts genaues, obschon sie über die Verteilung der beiden im lebenden Zustand qualitativ wie quantitativ nicht wenig besagen mögen (vgl. Fig. I u. 2). Davon kann man sich ohne Weiteres überzeugen, wenn man unter dem Mikroskop verschiedene Fixiermittel auf die lebenden Objekte einwirken lässt. Ich war dadurch imstande, des Näheren auf die Spur zu kommen, wie einerseits sich im Hyaloplasma durch Fällung, Entmischung usw. sichtbare Niederschläge verschiedener Formen bilden, während andererseits die Cytosomen (Mikrosomen) Verunstaltung, Verquellung, Aufösung, Verlegung oder Aggregation erleiden, bis schliesslich jene bunten Gefüge fixierten Zytoplasmas zur Ausbildung kommen.

Wie schon oben flüchtig angedeutet worden ist, stellen die Vakuolen, mit welchen das Zytoplasma auch der embryonalen, teilungsfähigen Zellen nicht selten beschickt ist, eine der launig fixierbaren Strukturen dar, weshalb man sie wohl ,, kolloidal “ zu benennen berechtigt sei. Sie zeigen sich für gewöhnlich optisch leer, wenn auch gewisse fixierbare Kolloide oft darin gelöst eingeschlossen sind. Bei Nekrobiosis ebenso wie bei der gewöhnlichen Fixierung findet Devakuolisation leicht statt, worauf aufs neue nachherige Vakuolisierung folgen kann, etwa wie BERG seinerzeit für Kernstoffe angesprochen hat."

Ausser Zellkern sind noch zwei Arten der metakolloidalen Strukturen im Zytoplasma beherbergt, nämlich Plastiden und Chondriosomen, auf welche ich hier nicht weiter eingehen werde.

Was eben erst von den Vakuolen gesagt worden ist, das gilt gerade auch für die in den erwachsenen Zellen die Safträume durchsetzenden Plasmastränge, welche sich beim Abtöten der Zellen schnell

I) Für das Nähere über die Vakuolen als Fixierungsartefakten verweise ich auf meine besondere Abhandlung. 
unter Einziehung zerreissen werden, was leicht begreiflich wird, wenn man sich an die von Lepeschkin ('24, S. 63; '25) neuerdings aufmerksam gemachte Tatsache erinnert, dass sie nur solange bestehen können, als die Plasmaströmung darin fortläuft. So finden wir wiederholt in fixierten Präparaten ringsum den Kern oder die Mitosenfigur herum ein gegen Saftraum hin mit einer hautschichtähnlichen Membran scharf umschriebenes Plasmastück hinterlassen. (Kerntasche HaNsteins '80). (Fig. 3 ). Beim flüchtigen Anblick täuscht ein solches Bild eine plasmolysierte Zelle oder einen doppelt behäuteten Kern vor.

Die im fixierten Zustand so auffällig erscheinende Kernwand erweist sich im Leben nicht immer wahrnehmbar. Bei den meisten beobachteten Pollenmutterzellen sowie den karyotinarmen Kern besitzenden Wurzelzellen (s. Yamaha und Shinotô '25) vermochte ich leider nicht die Kernmembran zuweilen mitsamt allen anderen Kernstrukturen lebend nachzuweisen. Erst beim Eintritt der Nekrobiosis werden sich die Einzelheiten der letzteren auch hier mit aller Schärfe unter den Augen enthüllen. Jedenfalls scheint die Kernmembran anderen Membranstrukturen völlig gleich zu sein. Es lässt sich im übrigen nicht so leicht entscheiden, ob und inwieweit die Angabe Schaedes ('25, S. 235, 237 usw.), dass auch bei anderen Wurzelzellen (Allium und Vicia) die ruhenden sowie sich teilenden Kerne für die Lebendbeobachtung ganz unzugänglich sein sollen, das Richtige trifft. Das hängt mit den Umständen zusammen, dass auch bei ein und demselben Objekt sich die Sichtbarkeit der Strukturen bis zu einem gewissen Grade nach der Untersuchungsmethode richtet und weiterhin dass man sich jeweilig zwischen dem gesunden und kranken bzw. normalen und anormalen Zustand der Zellen nicht in so einfacher Weise entscheiden kann. Es gelang mir in der Tat, wie man gleich unten sehen wird, in den unbedenklich gesund lebenden Zellen aus Wurzelspitzen verschiedener Pflanzen von einschlägigen Strukturen etwas zu entschleiern. Auf die Sichtbarkeit der Bilder scheinen der Quellungsgrad und Lipoidgehalt des Protoplasmas nicht geringen Einfluss zu haben, wofür unsere Fixierungsversuche sowie Lebendbeobachtung einige Anhaltspunkte abzustatten vermochten. Gesteigerter Hydratationsgrad und höherer Lipoidgehalt, demzufolge allenfalls höhere Viskosität des Protoplasmas machen die Einzelheiten der Strukturelemente lebend schwer unterscheidbar, so beispiels- 
weise bei den Wurzelzellen von Glycine Soja und Pollenmutterzellen überhaupt.

Nun wende ich mich einzelnen Strukturen des Zellkerns zu. Was zuerst das innere Gefüge des ruhenden und Interphasenkerns betrifft, so konnte ich die eigentliche Netzstruktur des Karyotins (Retikulum), wie sie gewöhnlich im fixierten Präparat vorkommt, nur ausnahmsweise mit Sicherheit feststellen, z.B. bei Sagittaria-Wurzeln.

Manchmal verteilt sich das Karyotinelement im Ruhekern in Tröpfchen gleichmässig fein und dicht, genau wie schon früher von LUNDEGÅRDH ('IOa, S. I77, I8I ; 'I2b, S. 240, 255) bemerkt wurde (Allium-, Vicia- und Pisum-Wurzeln, Tradescantia-Haare). (Fig. I).

In anderen Fällen, besonders bei karyotinarmen Kernen (Glycine-, Ricinus-, Isoetes-, Zea-Wurzeln usw.) sieht der ganze Kern optisch homogen aus, ${ }^{1)}$ nicht selten auch hier mit einer geringen Beimischung von flockigen Karyosomen (Fig. 4).

Im ersten Falle braucht einzelnen Karyotintröpfchen nicht eine kugelige Form zuzukommen, da jedes meistens zur Verzerrung dicht gegeneinander gedrängt ist, ohne jedoch zur gegenseitigen Verschmelzung zu kommen. „, Berührung ohne Verschmelzung “ gleichartiger Strukturelemente miteinander scheint gerade immer die Gesundheit derselben auszuzeichnen. Ebendasselbe wurde ferner häufig bei Chromosomen, Plastiden und Zytosomen wahrgenommen.

Für die Artefaktnatur der Netz-bzw. Anastomosenstruktur des Karyotinelements in fixierten Präparaten sprechen u. a. die Umstände,

I. dass die Anastomose des Karyotins als eine Folge des von Substanzverlust (auch Entwässerung) begleiteten „, Phasenumschlags “ aufgefasst werden kann, da sie sich im ersten Stadium der Nekrobiosis der Zellen sowie der karyotinauflösenden Wirkung ausgesprochen bemerkbar macht.

2. dass sie da unfehlbar mehr oder minder ausgeprägt erscheint, wo die sog. Membranstrukturen und sonstige Fixierungsartefakten mit Deutlichkeit zutage treten (vgl. Schaede '25, S. 247).

3. Und weiter, dass sie bei (nach Masse der naturgetreuen Bilder) besserer Fixierung immer schwerer nachweisbar zu werden pflegt.

I) Der Zellkein wurde bisher häufig als eine völlig homogene Struktur bezeichnet, z. B. von Frommann ('84, S. 28I), Chambers ('I4), Price ('I4, S. 616), Spek ('20), weiter vgl. Cowdry ('24, S. 265), SCimafde (loc. cit.). 
4. Man hat Grund anzunehmen, dass im Gegensatze zum Spirem und Chromosom, welch' beide ohne Zweifel den ,, metakolloidalen “ Strukturen entsprechen, die Karyotintröpfchen ebenso wie Mikrosomen eine von den ,, kolloidalen “ Strukturen der Zellen darstellen.

Angesichts dessen, was die fixierten und ungefärbten Objekte erschliessen lassen, erscheint unbestreitbar, dass die Netzstruktur des Karyotins auch durch die Färbung in ununterschätzbarer Weise verstärkt werden kann (Fig. 2 u. 3 ; vgl. LundeGÅRdH 'I 2a, S. $259 \mathrm{ff}$, Champy zit. nach Litardière '2 I, S. 274).

Bezüglich des Kernsaftes oder der Karyolymphe möchte ich hier nur darauf hinweisen, dass man sich bei den karyotinarmen Kernen (s. oben) von der optischen Leerheit desselben mit Leichtigkeit überzeugen kann. Wir dürften den Kernsaft wohl als das Gegenstück zum Hyaloplasma im Zytoplasma hinstellen, obgleich er sich von dem letzteren durch anscheinend höhere Dispersität und einigermassen schwächere Lichtbrechung auszeichnet, was möglicherweise der angeblichen Substanzarmut des Kerns im Vergleiche zum Zytoplasma zuzuschreiben ist. ${ }^{1)}$ Es bedarf meines Erachtens in dieser Hinsicht einer weiteren theoretischen Überlegung, weil eben darin die Bedingungen für die eigentümliche Empfindlichkeit gegen äussere Eingriffe und die verschiedenen Fixierungsbilder der Zellkerne zu liegen scheinen.

Es scheint mir keinem Zweifel mehr zu unterliegen, dass ein heller Hof um den Nukleolus, welchen wir in fixiertem Präparat (besonders bei sublimat-, salpetersäure- oder jodhaltigem Fixiermittel) zu sehen gewöhnt sind, eine Fixierungsartefakte ist. Das ähnliche Bild, wie es in vereinzelten Fällen in scheinbar lebenden Objekten $\mathrm{zu}$ bemerken ist, wird meines Wissens entweder durch die optische Täuschung oder durch die einsetzende Nekrobiose hervorgerufen (s. auch LUNDEGÅRDH 'i 2b, S. 256, LieHR 'i 7, S. I42, I47, Schaede '25, S. 238). Für das Entstehungsmoment des Nukleolushofes wird gemeinhin die Schrumpfung des Nukleolus bei der Fixierung angenommen. Demgegenüber habe ich schon anderswo

I) Es ist schon früher einigemal angegeben, dass der Zellkern im Gegensatze zum Zytoplasma von den verschiedenen leicht zur Entmischung führenden oder stark lichtbrechenden Stoffen frei sein soll, so z.B. anorganische Elektrolyte, Fette, Gerbstoffe. Kohlehydrate, Farbstoffe usw. (vgl. Collip '20, Pratje '20, Tschermak '24, S. 585). 
die Meinung geäussert, dass die betreffende Hofbildung vielmehr wesentlich durch die Karyotinaushöhlung infolge des Substanzverlustes seitens des Karyoplasmas (als ein gewisses Stadium der Nekrobiosis oder auch bei künstlicher Auflösung des Karyotins usw.) bedingt werden soll (vgl. Yamaha und Sinotô '25, S. 2 I2 und weiterhin VAN Wisselingh '21, S. 305, Litardière '2 I, S. 372), eine Ansicht, welche sich auf folgenden Tatsachen stützt:

I. Die Messung des Durchmessers von hofhaltigen sowie hoffreien Nukleolen ergibt überzeugenderweise, dass in den meisten Zellen der Nukleolus durch die Hofbildung tatsächlich keine entsprechende Volumverminderung erleidet.

2. Der Nukleolushof tritt bei der unvollkommenen Fixierung ausgeprägt hervor, und zwar zumal da, wo die Membranstrukturen, das Karyotinnetz und die Zytoplasmaschrumpfung auffällig erscheinen.

3. Die Hofbildung wird mehrfach durch die niederen Konzentrationen von den karyotinauflösenden Stoffen (s. Yamaнa und Sinotô '25, S. 214) bewirkt.

4. Bei der Wirkung derjenigen Substanz, welche unbezweifelt dis Zusammenziehung des Nukleolus hervorruft, kann der letztere noch hoffrei bleiben, wofern nur das Karyotin dabei keiner Auflösung anheimfällt.

5. Der Nukleolushof ist bei den karyotinreichen Kernen (z.B. Allium, Sagittaria) recht selten bemerkbar, während bei den karyotinarmen (z.B. Ricinus, Glycine, Isoetes) die Hofbildung der Regel nach bei der Fixierung so gut wie unausbleiblich $z u$ sein scheint (vgl. Yamaha und Sinotô '25, S. 212).

6. Es wäre kaum denkbar, dass der Nukleolus, dem eine ungleich gleichmässigere und dichtere Konsistenz zukommt, durch die Reagenzien leichter schrumpfen kann, als das kolloidal viel gröber strukturierte Karyotin.

Im Leben bleibt der Nukleolus bisweilen ganz unsichtbar, so z.B. bei Glycine-, Ricinus-Wurzeln, Pollenmutterzellen von Tradescantia. In den ruhenden sowie Interphasenkernen der embryonalen Zellen, falls er einmal gelegentlich zu Gesicht kommt, zeigt er sich stets vollkommen sphärisch, etwa wie ein Öltropfen im Zytoplasma, auch wenn der Kern ziemlich von der Kugelform abweicht (wie bei Sagittaria-Wurzeln). Bei dem karyotinreichen Kerne ist es Regel, dass die Karyotintröpfchen so eng an die Nukleolusoberfläche anhaf- 
ten, dass die letztere mitunter sogar stachelig erscheint, genau wie es auch in fixierten Präparaten häufig auffällt. Solch einen an den Nukleolus festgeklebten Karyotinanteil findet man vereinzelt zum Teil auf der Stelle zurückgelassen, wenn bei der Fixierung ein Hof um den Nukleolus gebildet werden soll. Das Innere des Nukleolus scheint von einer recht homogenen Struktur zu sein, wenn er nicht, wie es bei den Glycine-, Sagittaria- und Vicia-Wurzeln und Pollenmutterzellen einiger Pflanzen häufig der Fall ist, mit den Vakuolen durchsetzt ist, welche ohne $Z$ weifel auch durch die Fixiermittel (besonders salpetersäurehaltige) erweitert oder neugebildet werden können. Die Membranstruktur um den Nukleolus lässt sich für gewöhnlich nicht so auffallend nachweisen wie sonst, was möglicherweise auf die leichte Konservierbarkeit (Fällbarkeit) oder schwere Exosmierbarkeit (Auflösbarkeit) der Nukleolarsubstanz und weiterhin auf einen besonderen kolloidalen Zustand derselben schliessen lässt. (s. Yamaha und Sinoto '25, S. 2 I4). ${ }^{\text {) }}$

Wenn der Kern sich zur Teilung anschickt, so nehmen die Karyotintröpfchen, unter Vermehrung der Lichtbrechung, durch ihre Hydratisierung und stellenweise Zusammenfliessung immer mehr an Volumen zu. Die Wasseraufnahme durch Kernsubstanz in der Prophase der Mitose äussert sich weiter auch in der Volumvergrösserung des ganzen Kerns (vgl. Schaede '25, S. 250). Auch im Dunkelfelde zeigt sich der Prophasenkern dementsprechend unverkennbar dunkler bzw. minder leuchtend als der Ruhekern. Die Spirembildung scheint also durch die Verschmelzung derart gequollener Karyotintröpfchen in bestimmter Anordnung (s. SchaEde '25, S. 250) mit gleichzeitiger Verdrängung der Substanz nach der Grenzfläche hin bewerkstelligt $z u$ werden, wodurch die Spireme oft gleichsam ein dünn aber deutlich gesäumtes Aussehen bekommen (Fig. 5). Das letzte Verhältnis tritt in ausgebildeten Chromosomen noch auffälliger hervor (s. unten).

Die angebliche Amöboidie des Nukleolus in Prophase, welche im fixierten Objekt widerholt $z u$ beobachten ist (besonders bei Allium-Wurzeln; s. auch Yамана und Sinotô '25, S. 206), konnte

I) Die etwas absonderliche Struktur des Nukleolus, die von Wager ('o4) usw. bei anderen Materialien bemerkt ist, fällt augenscheinlich in eine andere Kategorie der Fixierungsbilder, die andererorts Erwähnung finden soll. 
ich in lebenden Zellen leider nicht mit Sicherheit konstatieren (vgl. Chodat '24).

Die Polkappen, wie sie gewöhnlich als ein allgemeines Vorkommnis in der späteren Prophase bei Wurzelzellen angesehen sind, wurden bei den karyotinarmen Kernen sowie Haarzellen niemals, auch bei Vicia- und Allium-Wurzeln nur vereinzelt angetroffen, wobei sie etwa wie Vakuolen homogen erscheinen (s. LundEGÅRDH 'I2b, S. 249).

Es fällt uns gerade in diesem Stadium eine Anhäufung von Mikrosomen auf, welche sich an jede Polseite der Kernoberfläche ansammeln (Fig. 5). Dieses Polplasma (vgl. z.B. Nemec 'O I, S. 46, 48, Lundegrådh 'ize, S. 473ff, Yamaha '2Oa, Robyns '24, S. 434, Schaede '25, S. 252, Strasburger '84, S. IO9) bleibt im weiteren Verlaufe der Mitose an den Teilungspolen beibehalten, bis es zum Ende der Anaphase plötzlich verschwindet, um schliesslich an der Äquatorialebene wieder $z u$ erscheinen (s. Strasburger loc. cit). ${ }^{1}$

Bemerkenswert sei noch, dass in der späteren Prophase der Kernsaft merklich stärker lichtbrechend geworden ist als früher. Im fixierten Objekt findet man ihn häufig erst in diesem Stadium schwach homogen gefärbt (vgl. z.B. Litardière '2 I, S. 293, 306, 379).

Das Verschwinden der Kernmembran scheint ganz plötzlich einzutreten (bei Haarzellen). Wiederholt ist mir ein solches Bild begegnet, das den Eindruck machen konnte, als ob die Kernwand dabei einer körnigen Degeneration unterliegen möchte. Der umgekehrte Vorgang d.i. die direkte Verschmelzung der Mikrosomen zur Bildung der Kernwand fiel schon früher FrommanN ('84, S. 42) auf.

Es ist von Interesse $z u$ bemerken, dass eben darauf ein Stadium einfällt, wo das ,, tassement “ der Chromosomen stattfindet, und zwar dass die ganzen Chromosomen ohne die geringste Miteinanderverschmelzung gewissermassen $\mathrm{zu}$ einem Klumpen zusammengedrängt werden. Diese Zusammenballung der Chromosomen, der ich früher nach Analogie von ,, tassement polaire “ GRÉGolRES ,, tassement équatoriale" beigelegt habe, kann durch Fixiermittel und auch unter

I) Bei abnorm höheren Temperaturen hebt sich das Polplasma gegen das umgebende vakuolenreiche Zytoplasma scharf hervor, so dass wir weiterhin verfolgen kōnnen, was daraus wird. Es ist aber hier nicht am Platze, auf die genetische Beziehung zwischen Pol- und Äquatorialplasma näher einzugehen (s. meine weitere Mitteilungen). 
verschiedenen ungünstigen Bedingungen verstärkt oder vorgetäuscht werden (für Näheres s. weitere Mitteilung) (Fig. 6). Das ähnliche kommt auch bei dem Wiedererscheinen der Kernwand in Telophase vor (s. unten).

Im Leben sind das ausgebildete Spirem und Chromosom innen ganz homogen (optisch leer) strukturiert, und aussen mit einer mässig dicken im Dunkelfelde etwa körnig erscheinenden Membran bekleidet. Diese Aussenhülle der Chromosomen vertritt ohne Zweifel eine der unfixierbaren Naturstrukturen und entspricht demgäss nicht derjenigen Membranstruktur, welche in fixierten Präparaten mit Häufigkeit um jedes Chromosom in einem beim flüchtigen Anblick verwechselnd ähnlichen Aussehen auftritt. Hier sei noch hinzuzufügen, dass der Pollenmutterzelle von Tradescantia in heterotypischer Meta- und Anaphase eigentümlich strukturierte (metakolloidal ?) Chromosomen zukommen (Fig. 8); Ein jedes besteht. nämlich aus dem stärker lichtbrechenden Mark und der dunkler erscheinenden Rindenpartie. In sonstigen Fällen ist irgendeine Struktur (metakolloidal) der Chromosomen, soweit meine Beobachtung reicht, in gesunden Zellen ganz ausgeschlossen (vgl. Schaede '25, S. 25 I).

Der Chromosomenkomplex wird in einem stark lichtbrechenden, optisch leeren Raum eingebettet. Dieser sog. Spindelraum (LundEGÄRDH 'I2b, S. 250 ; 'I2c, S. 380 usw.) wächst bis gegen das Ende der Anaphase immer an seiner Lichtbrechung und bleibt von allerlei körnigen Elementen (ultramikroskopisch wie mikroskopisch auflösbaren dispersen Phasen, z.B. Mikrosomen, Cytosomen usw.) völlig befreit, welch letztere häufig in Form eines Mantels den Spindelraum umwallen.

Solange die Karyokinese und Zytokinese normal ungestört verlaufen können, lässt sich von den sog. achromatischen Fäden (Spindelfäden, Verbindungsfäden) darin nichts unterscheiden. Das Gegenteil finden wir aber vereinzelt in der Literatur selbst bis in die neueste Zeit angegeben (vgl. z.B. Tischler '2I-'22, S. 349, MainX '24, S. 556, 557, Schaede '25, S. 24I). Auf Grund eigener Erfahrungen müssen wir jedoch in solchen Fällen die Gesundheit des beobachteten Objektes in Verdacht stellen (s. Chodat '24). Ob an der Sichtbarkeit entweder das Nichtvorhandensein oder das Lichtbrechungsverhältnis Schuld ist, darüber kann man sich von morphologischer Seite allein aus nicht ohne Weiteres entscheiden. Nach unseren 
Fixierungsversuchen ist es andererseits höchst wahrscheinlich geworden, dass der nur in fixiertem Zustand geläufig bekannte achromatische Faden nichts anderes darstellt als eins von den Fixierungsbilder (Fällungsformen) der Spindelsubstanz, welche genau wie etwa jene filzige Struktur des Zytoplasmas unter bestimmten Bedingungen der Zellen zum Ausdruck kommen können, was sich anschaulich mit jeweiligem kolloidalen Zustand einzelner Strukturen (hier vermutlich Quellungszustand) verknüpft. Somit befremdet es uns nicht im geringsten mehr, dass meine an einem anderen Orte noch aufzuführenden experimentell-zytologische Untersuchungen zu einem bemerkenswerten Rückschlusse geführt haben, und zwar dass die fädige Struktur der Spindelfigur nichts mit dem normalen Verlaufe der Zytokinese, umsomehr der Karyokinese zu tun hat. Es kann also nicht mehr in Frage kommen, dass die achromatischen Fäden ein Zellorgan (metakolloidale Struktur) vertreten, an dessen Hand die regelmässige Verteilung der Chromosomen erst durchgeführt werden kann, eine Ansicht, welche noch in den letzten Jahren von einigen Autoren (z.B. Herrig ('22), Mainx ('24)) verteidigt wurde. Überraschend sei weiter, dass die ähnliche faserige Struktur durch die sehr verdünnten Säuren (z.B. $\mathrm{HCl}$ von $\mathrm{PH}_{3}, 8-4,0$ bei TradcscantiaHaaren) reversibel sichtbar gemacht werken kann (vgl. Cowvdr '24, S. 407, Lewis '23), wobei die Mitosen vorübergehend zum Stillstand kommen. Bezüglich der Artefaktnatur der achromatischen Fäden muss hier noch hervorgehoben werden, dass sich die Deutlichkeit desselben Bildes in fixierten Präparaten unter anderem nach dem Exosmosegrade der Zellsubstanzen richtet. Die Ansicht wird durch folgende Befunde bestärkt.

I. Die Spindelfigur lässt sich nur mit sauren Fixiermitteln von $\mathrm{PH}_{\mathrm{H}}<\mathrm{I}, 4$ faserig fixieren, niemals mit solchen von $\mathrm{PH}_{\mathrm{H}}<2,4$, vorausgesetzt, dass eine einwandfreie Fixierung vollzogen worden ist.

2. Die lipoidlöslichen Stoffe, wie z.B. Alkohole, Äther, Chloroform vermögen die fädige Struktur der Spindelsubstanz zu verdeutlichen.

3. Die Deutlichkeit der Spindelfäden gehen in demselben Präparate immer mit deren der Membranstrukturen (s. auch unten) genau parallel.

4. Bei erhöhter Temperatur und durch die Wirkung der Hypertonie erscheinen die achromatischen Figuren dem substanzarmen Zytoplasma gegenüber ausgeprägt. 
5. Bei je deutlicherer faseriger Struktur der Spindelsubstanz wird die dazwischen liegende homogen, oder genauer homogengerinnselig färbbare Substanz (vgl. Yamaha ('20a u.b), Timberlake ('oo), Robyns ('24, S. 393, 42 I, 422, 436 usw.) um so spärlicher.

Nachdem die Tochterchromosomen an die Pole gelangt sind, drängen sie sich so eng zusammen, dass zwischen jeden kein freier Raum übrig gelassen $\mathrm{zu}$ sein scheint (tassement polaire GrÉGOIREs) (Fig. 7). Dabei ist eine völlige oder sogar teilweise Verfliessung der Chromosomen ganz ausgeschlossen, wie sie in fixierten Objekten als eine Artefakte nicht selten auftritt. Im nächsten Augenblick finden wir eine neue Kernmembran wahrscheinlich von der Seite des Zytoplasmas her dicht auf die Aussenseite des „, tassement “ angelegt. Dieser Vorgang lässt sich bei Haarzellen in vivo aufs klarste verfolgen. Die Wiederherstellung der Kernwand scheint zeitlich angenähert mit dem Verschwinden des Polplasmas übereinzustimmen (vgl. oben). Eben in diesem Stadium wird der maximale Abstand zwischen beiden Tochteranlagen erreicht, der, wie schon früher bei fixierten Wurzelzellen bemerkt worden ist (s. YAMAHA '2Oa, weiterhin HABERMEHL 'o9), in normaler Zelle eines gleichartigen Gewebes aus derselben Pflanze immer genau gleich ausfällt. In der früherer Telophase konnte ich ausser einigen zweifelhaften Fällen sowohl die Anastomosen als Vakuolisierung der Chromosomen nirgendswo bemerken. Es hat vielmehr den Anschein, dass die Karyotinelemente durch die tropfige Entmischung (?) aus Chromosomensubstanz, deutlich in der äusseren Partie (Aussenhülle) jedes Chromosoms wiederhergestellt werden, wobei zweifelsohne die Membranstruktur des letzteren (Phasengrenzschicht) damit an ihrer Beständigkeit einbüssen muss, was natürlicherweise einen bedeutsamen Rückschluss bedingt; nämlich dass die in fixiertem Zustand anzutreffenden Chromosomenanastomosen sowie Vakuolisation auch in Telophase eine offenbare Artefakte sind, die aller Wahrscheinlichkeit nach mit dem gelegentlich eingetretenen, besonderen kolloidalen Zustand der Chromosomen zusammenhängt. Über die Längslichtung der Chromosomen in Telophase konnte ich wie in Prophase leider nichts genaues ermitteln.

Bei fixierten Materialien wird die erste Anlage der Scheidewand, erst gerade in dem Stadium von „, tassement “, in der Mitte der Teilungsebene, entweder als die äquatoriale Verdickung der Verbindungsfäden (bei den sauren Fixiermitteln von $\mathrm{P}_{\mathrm{H}}<2,4$ ), oder falls 
die letzteren unsichtbar sind, einfach als eine zusammenhangende Körnerplatte angedeutet (vgl. Yамана '2Oa u.b ; '2 I-'22). Zu meiner Überraschung fande ich aber kein körniges Element in diesem Stadium an der Äquatorialebene des Phragmoplasten im lebenden Objekt, sondern noch später waren die nicht genau in einer Reihe gestellten, also den Dermatosomen in fixierten Objekten nicht entsprechenden Mikrosomen kaum dann erkennbar gewesen, als eine zarte stark lichtbrechende Linie (junge Zellwand?) an der Äquatorialebene erschien. Von der Doppelnatur der jungen Hautschicht, wie sie früher angenommen wurde (Yамана '2Oa u.b), kann man sich erst nur durch die Plasmolyseversuche sowie in fixiertem Zustand (s. meine experimentell-zytologische Untersuchungen) überzeugen (s. auch Yamaha '2I-'22). Im lebenden Objekt gelang es mir also nicht einmal die körnigen Elemente der Zellplatte (Dermatosomen) oder selbst irgendeine zusammengeschlossene Körnerschicht festzustellen. Nach obigem ergibt sich, dass die sog. Zellplatte, wie sie in fixiertem Zustand mit Deutlichkeit in Erscheinung tritt, ebensowohl eine Fixierungsartefakte darstellen kann wie die Verbindungsfäden selbst, da die beiden sich in gleicher Weise der Lebendbeobachtung unzugänglich erwiesen haben (s. auch oben). ${ }^{1)}$

Schon längst ist häufig die Angabe geltend gemacht worden, dass die kinoplasmatischen Fäden, namentlich die Verbindungsfäden eine schon lebend sichtbare Struktur sein sollen. (s. oben, für ältere Literatur vgl. Yамана '2I-'22; '25a). Dieser Sachlage scheint ein Beobachtungsfehler zugrunde zu liegen, der darauf beruht, entweder dass der Spindelraum insbesondere im Kerntonnenstadium leicht zur fädigen Fällung geneigt ist (demzufolge in krankem Zustand), oder dass die Polstrahlung, Plasmastränge und selbst Chromosomen in vivo häufig die betreffende faserige Struktur vortäuschen können und infolgedessen fälschlich dafür gehalten wurden. Dass nach SchaEde ('25, S. 24I) die angeblichen Verbindungsfäden beim Zellentode verschwinden sollen, wäre aus den oben angedeuteten Tatsachen ersichtlich, desgleichen dass die fadige Fällung der Spindelsubstanz in saurem Medium auch reversibel vorgehen kann und weiter dass

I) Der ausführlichen Erörterung über Zytokinese im allgemeinen ist eine andere Abhandlung gewidmet. 
die letztere beim allmählichen Absterben der Zellen als eine Quellungsform eine Homogenesierung erleiden kann (vgl. meine weitere Mitteilung).

Nicht wenige Forscher setzen sich für die Ansicht ein, dass die Verbindungsfäden dem Wesen nach von den Spindelfasern ganz verschieden sein sollen (s. Yамана '20a, '25a; weiter Postma 'o9, Stomps 'io, 'i i, Robyns '24, S. 388, 436, Tischler loc. cit.). Dieser Behauptung, die nur unter der Annahme, dass die achromatischen Fäden auch im Leben wirklich als solche existieren, standhalten kann, liegen eine Reihe mikroskopischer Befunde zugrunde; z.B.

I. Die Spindelfasern bei Karyokinese erfahren gegen das Ende der Anaphase eine vollständige Rückbildung, bevor die Verbindungstäden (fuseau de cytocinèse Robyns') zwischen beiden Tochteranlagen ausgespannt erschienen sind.

2. Die Verbindungsfäden sehen, wie es Regel ist, dem Fixierungsbild nach gegenüber den Spindelfäden etwas verschieden aus.

3. Im Gegensatze zu den Spindelfasern können die Verbindungsfäden auch in vivo sichtbar sein.

Allem obigen gegenüber ergaben sowohl meine eigene Beobachtungen an fixierten und lebenden Objekten als auch die experimentellen Untersuchungen gar nichts, das sich zugunsten der Meinung, dass die Verbindungsfäden unabhängig von der Kernspindel nachher aufs neue gebildet werden sollen, verwerten liesse. Abgesehen davon, dass die faserige Struktur der Spindelsubstanz (achromatische Sub$\operatorname{stan} z$, Kinoplasma), wie schon oben angedeutet, möglicherweise auf nichts anderes als eine Fixierungsartefakte hinausläuft, hat man im Gegenteil vollen Grund, die genetische Beziehung zwischen Spindelund Verbindungsfäden anzunehmen. In lebender Zelle bleibt die Spindelsubstanz vom Anfang der Entstehung an (d.i. Polkappenstadium) bis zur Zeit, wenn die erste Anlage der Scheidewand in Erscheinung tritt, den gegen das umgebende Zytoplasma ziemlich scharf abgehobenen, glashell homogen aussehenden Zustand, mit immer zunehmendem Lichtbrechungsverhältnis, unausgesetzt beibehalten. Nach Fixierung, zumal bei der Anwendung von sauren Fixiermitteln, wo sich die achromatische Substanz mehr oder minder faserig erkennen lässt, vermehren sich die betreffenden fädigen Elemente in Anaphase und Telophase immer, anstatt sich irgendeiner 
Rückbildung zu unterwerfen, sofern die Zellplattenbildung daraufhin folgen soll (Yамана '2Oa und $b$ ). ${ }^{1)}$

Nach meinen experimentell-zytologischen Untersuchungen verhalten sich die achromatischen Fäden sowohl in Metaphase wie in Anaphase und Telophase verschiedenen äusseren Eingriffen gegenüber in ganz derselben Weise, ausser einigen Ausnahmesfällen, wo der eintretende Reaktionsunterschied auch nicht über einen quantitativen hinaus geht. Auch auf dem mikrochemischen Wege lässt sich ebenfalls die Zusammengehörigkeit beider Strukturelemente erschliessen, nämlich beispielsweise in Bezug auf die Widerstandsfähigkeit gegen rauchende Salzsäure, Eau de Javelle, heisses Wasser usw.

Beiläufig möchte ich hier noch die Frage berühren, ob die achromatischen Fäden gebührend als ein Vakuolensystem aufzufassen sind, wie bislang häufig getan wird, so z.B. von Densmore ('o8, S. 306, 309), Postma ('o9), Stomps ('i i, S. 292ff), Devisé ('22), Zimmermann ('23, S. I 38 , I45), Robyns ('24, S. 376), Nienberg ('24, S. 98), Rosen (25, S. 213, 214), usw. Die Deutung des Spindelfadens als Vakuolenwand erscheint zwar sehr verlcckend, um so mehr, als nach unseren Fixierungsversuchen sich die Spindelfäden in mancher Hinsicht genau wie eine Membranstruktur (s. oben) verhalten, aber hinsichtlich seiner Beeinflussung durch verschiedene Agenzien wie seines mikroskopischen Bildes (Fixierungsbild) weicht das sog. Kinoplasma tatsächlich so augenscheinlich von den Plasmasträngen oder Vakuolen selbst $a b$, dass die angesprochene Gleichsetzung beider Strukturen nichts mehr als ein theoretisches Interesse verdient, sofern den Vakuolen der gewöhnliche Sinn des Wortes beigemessen wird, denn,

I. Die kinoplasmatischen Fäden als ein Komplex langgezogener Vakuolen hinzustellen, heisst gerade ihre Realität wegzuleugnen, indem man dieselben den Plasmasträngen zur Seite stellt, was offenbar den Befunden meiner Lebendbeobachtung widerspricht.

2. Die achromatischen Fäden als ein Fixierungsbild zeigt sich den verschiedenen Fixiermitteln gegenüber merkwürdigerweise beständig, während die Vakuolen und Plasmastränge bei der Fixierung leicht der Zerstörung ausgesetzt werden (s. oben).

r) Es geht allerdings bei der Pollentetradenbildung der simultanen Zellteilung eine Rückbilelung der achromatischen Fäden voraus, und zwar nicht seitens der Spindelfäden, sondern seitens der Verbindungsfäden mitsamt den Zellplattenelementen (vgl. Yamaha '20a, b). 
3. Die Spindelsubstanz und die Vakuolen reagieren auf die verschiedenartigen Eingriffe (chemische, thermische oder mechanische) in gründlich abweichender Weise. Für einzelnes Tatsachenmaterial verweise ich auf meine weitere Mitteilungen.

Zum Schlusse möchte ich noch Herrn Prof. Dr. K. Fujır für seine freundliche Unterstützung bei dieser Arbeit meinen herzlichsten Dank aussprechen. 


\section{LITERATURVERZEICHNIS.}

BechHold, H. (1920) Die Kolloide in Biologie und Medizin, Dresden u. Leipzig, 3. Aufl.

BeEr, R. (1906) Beih. z. bot. Ctbl. I. Abt. I9: 286-313.

Berthold, G. (I886) Studien über Protoplasmamechanik, Leipzig.

Chambers, R. (I914) Science, 50; 824-827.

Chodat, R. (1924) Cpt. R. Soc. Phys. et Hist. nat. Genève, 41 : 96-99.

Collip, J. B. (I920) Journ. of Biol. Chem. 42; 227-236.

Cowdry, E. V. (1924) General Cytology, Chicago.

Densmore, H. D. (1908) Univ. of Cal. Publ. in Bot. 3; 303-330.

Devisé, R. (I922) La Cellule, 32; 247-309.

Frommann, C. (I884) Jena. Ztschr. f. Naturw. I7 ; I-349.

Habermehl, K. (I909) Die mechanischen Ursachen für die regelmässige Anordnung der Teilungswände in Pflanzenzellen, Dissert. München.

Hanstein, J. v. (I880) Das Protoplasma, Heidelberg.

Herrig, F. (I922) Beitr. z. allgem. Bot. 2; 403-4II.

Hofmeister, W. (I897) Die Lehre von der Pflanzenzelle, Leipzig.

KüHne, W. (I864) Untersuchungen über das Protoplasma und die Contractilität, Leipzig.

Kunstler, J. et Prívost, F. (1924) La matière vivante, Paris.

Küster, E. (1924) Experimentelle Physiologie der Pflanzenzelle, Abderhaidrns Handb. d. biol. Arbeitmeth. Abt. XI, Teil I, Heft 7.

Lepeschkin, W. (I924) Kolloidchemie des Protoplasmas, Berlin.

$\longrightarrow$ (1925) Ber. d. D. bot. Ges. 43 ; 2 I-26.

Lewis, m. R. (1923) Buil. of Johns Hopkins Hosp. 34 ; 373-379.

Lidforss, B. (I9I5) Allgem. Biol. Die Kultur der Gegenwart 3. Teil, 4. Abt. I. Bd. S. $218-264$.

Liehr, O. (I9I7) Cohns Beitr. z. Biol. d. PH. I3: I35-220.

Litardière, R. de (I92I) La Cellule, 31 ; 255-473.

LunDEGÅRDiI, H. (I9IO) Svensk Bot. Tidsk. 4: I74-I96.

-, (I912a) Archiv für mikr. Anat. 80; 223-273.

$\longrightarrow$, (1912b) Jahrb. f. wiss. Bot. $51 ; 236-282$.

$\longrightarrow$, (I9I2c) Cohns Beitr. z. Biol. d. Pfl. I I : 373-542.

- (I922) Zelle und Zytoplasma, Berlin.

Mainx, F. (1924) Zool. Jahrb. Abt. f. allgem. Zool. u. Physiol. d. Tiere 4I ; 553580 .

Miehe, H. (I90I) Flora 88; 105-142.

NemeC, B. (I9OI) Beitr. z. wiss. Bot. 4; 37-92.

NienberG, W. (1924) Ber. d. D. bot. Ges. 42; 95-99.

Osterhout, W. J. V. (I9I3) Bot. Gaz. $55 ; 446-45$ I.

Ostwald, Wo. (I9I9) Die Welt der vernachlässigten Dimensionen, I)resden u. Leipzig, 3. Aufl.

Pfeffer, W. (I92I) Osmotische Untersuchungen, 2. Aufl., Leipzig.

Postma, G. (I909) Bot. Ztbl. II4; 297 ; I9Io (Ref.).

Pratje, A. (I920) Biol. Ctbl. 40: 88-iI3. 
Price, R. (I9I4) Ann. Bot. 28; 60I-632.

Robyns, W. (1924) La Cellule 34; 367-454.

Rosen, F. (I925) Ber. d. D. bot. Ges. 43; 21I-2I7.

Sakamura, T. u. Loo, T.-L. (1925) Bot. Mag. Tokyo 39: 6I-76.

Schaede, R. (1925) Cohns Beitr. z. Biol. d. Pfl. I4; 231-26o.

Schneider, E. (I925) Zeitschr. f. wiss. Mikr. 42; 32-54.

Schwarz, Fr. (I892) Cohns Beitr. z. Biol. d. Pfl. 5 ; I-244.

SeIfriz, W. (I92I) Ann. Bot. 35; 269-296.

Spek, J. (1920) A rchiv. f. Entw.-Mechan. 46: 537-546.

Stomps, Tir. J. (I9Io) Kerndeeling en Synapsis bij Spinacea oleracea, L. I)iss. Amsterdam.

, (I9II) Biol. Ctbl. 31; 257-309.

Strasburger, E. (I884) Die Controversen der indirecten Kerntheilung, Bonn.

-, (I888) Histol. Beitr. Heft I. Jena.

Timberlake, H. G. (1900) Bot. Gaz. 30; 74, I54ff.

Tischler, G. (I92I-I922) Allgemeine Phanzenkaryologie, Berlin.

Tschrrmak, A. v. (I924) Allgemeine Physiologie, Bd. I. Berlin.

DE Vries, H. (1885) Jahrb. f. wiss. Bot. I6; 465-598.

Wager, H. (1904) Ann. Bot. 18; 29-55.

Weis, A. (1925) Arch. f. wiss. Bot. I : $145-186$.

Wrlson, E. B. (I924) in Colvdry's (ieneral Cytology, Chicago.

Van Wissfilingir, C. (I92I) Beih. z. bot. Ztbl. I. Abt. 38; 273-3j4.

Yamain, G. (I9zoa) Bot. Mag. Tokyo, 34 ; (199)-(212).

-, (1920b) Ebenda 34; I I7-129.

$\longrightarrow$, (192I-I922) Gendai no Kwagaku Io: 21I-224, 289-315, 365-38I, 4II-423, $452-460$.

- (1925a) Bot. Mag. Tokyo, 39; (36)-(41), (69)-(76), (103)-(116), (132)-(140).

——, (1925b) Ebenda 39; (164)-(167).

Yamaha, G. u. Sinotô, Y. (I925) Ebenda, 39; 205-219.

Zacharias, E. (I888) Bot. Ztg. I888, S. 33, $5 \mathbf{I}$.

—, (1910) Progr. rei. bot. 3; 67-258.

Zimmermann, W. (I923) Zeitschr. f. Bot. I5; II $3^{-175}$.

\section{Figurenerkl.̈̈rUng.}

Alle Figuren, die sich mit Ausnahme von Fig. 2 u. 3, welche den mit FLEM MINGscher Fixierungsflüssigkeit (Bonner Rezept) fixierten Objekten entnommen wurden, auf lebende Materialien beziehen, wurden unter Hell- und Dunkelfeld mit Hilfe von ABBsschem Zeichenapparate, unter Anwendung von ZeIss' Apochromat-Objektiv $2 \mathrm{~mm}$ (N.A. I, 3) und Kompensationsokular 8 ( $\times$ ro) gezeichnet. Vergrōsserung ca. II70 (Fig. I-6, u. 8) oder ca. 600 (Fig. 7).

Fig. I-3, Vicia Faba (Wurzelspitzenzellen).

Fig. 4, Ricinus communis (Wurzelspitzenzelle).

Fig. 5, Zelle aus Schuppenhaare von Polypodium sp.

Fig. 6, Allium Cepa (Wurzelspitzenzelle).

Fig. 7, Tradescantia virginica (Zelle aus Staubfadenhaare).

Fig. 8, Tradescantia airginica (Pollenmutterzelle). 